\title{
Production of DUSP1 protein using the baculovirus insect cell expression system and its in vitro effects on cancer cells
}

\author{
PENG CHENG*, SHUYING ZHU*, LI JUN, LIHUA HUANG and YAHUI HONG \\ College of Bioscience and Biotechnology, Hunan Agricultural University, Changsha, Hunan 410128, P.R. China
}

Received October 12, 2014; Accepted April 6, 2015

DOI: $10.3892 / \mathrm{ijmm} .2015 .2179$

\begin{abstract}
The aim of the present study was to produce the human dual specificity phosphatase 1 (DUSP1) protein with biological activity and to investigate its in vitro effects on cancer cells. DUSP1 protein was expressed in the baculovirus expression system and purified by Ni-affinity chromatography followed by dialysis in PBS. The purified protein was verified by SDS-PAGE and western blot analysis. Six cancer cell lines were then cultured in the presence of DUSP1 for various periods of time, and the phosphorylated extracellular signal-regulated kinase ( $\mathrm{p}-\mathrm{ERK}$ ) content in each cell line was subsequently determined by western blot analysis. Compared to the $\beta$-actin level, the amount of p-ERK markedly decreased after $1 \mathrm{~h}$, indicating that DUSP1 suppressed the expression of p-ERK in 6 cancer cell lines examined. Human cervical cancer cells were also collected and counted following co-culture with DUSP1 to examine its effect on the growth rate of cancer cells. A baculovirus expression system for the production of DUSP1 protein was successfully constructed. The p-ERK content was found to be significantly decreased when the cancer cell lines were exposed to DUSP1. The capability of binary fission was reduced when the cells were examined under a microscope. The proliferation of human cervical cancer cells was also inhibited by DUSP1.
\end{abstract}

\section{Introduction}

The dual-specificity phosphatase enzyme belongs to the protein tyrosine phosphatase (PTP)/dual specificity phosphatase (DSP) family. It is involved in numerous basic physiological activities, including cell signaling, chondrocyte growth and other cell metabolic processes. The majority of DSPs play a key role in the regulation of mitotic signaling and cell cycle control following

Correspondence to: Dr Yahui Hong, College of Bioscience and Biotechnology, Hunan Agricultural University, 1 Nongda Road, Furong, Changsha, Hunan 410128, P.R. China

E-mail: yahuihong@vip.sina.cn

*Contributed equally

Key words: dual specificity phosphatase 1, Bac-to-Bac, expression, purification, phosphorylated extracellular signal-regulated kinase external stimulation (1-5). The activity of mitogen-activated protein kinase (MAPK) is suppressed following its dephosphorylation by dual specificity phosphatase 1 (DUSP1). A previous study demonstrated that DUSP1 not only inhibits the activity of MAPK3/1, but also regulates the expression of its CG subunit (6). Nyati et al (7) reported that telangiectasias caused by radioactive mutation downregulates the function of phosphor-extracellular signal-regulated kinase (p-ERK) and accordingly enhances the activity of DUSP1. Wang et al (8) found that DUSP1 expression was induced by the abnormal expression of the transcription factor, E2F-1, thereby providing a link between E2F-1 and MAPK. During septicaemia, DUSP1 minimizes damage to the liver (as well as other organs) by suppressing the levels of pro-inflammatory cytokines (9). DUSP1 has also been shown to inhibit the growth of liver cancer cells through the regulation of ERK (10), and its expression has been shown to be regulated by cancer related genes, such as p53 (11). It has also been previously demonstrated however, that the level of DUSP1 in the blood correlates with post-operative morbidity and that high levels of DUSP1 cause prolonged hospitalization following coronary artery bypass grafting (12). Cancer cells are characterized by uncontrolled cell division, primarily due to the continued activation of MAPK. Since DUSP1 phosphorylates MAPK, it has the potential to modulate cancer cell proliferation. However, to the best of our knowledge, this has not been investigated to date due to the difficulty in expressing the protein with biological activity.

The baculovirus expression system has recently been identified as a superior tool compared with the Escherichia coli (E. coli) system due to its simple operation procedures, the high expression of co-factors and the close-to-natural function of the expressed proteins (13). Similarly, the Bac-to-Bac expression system, a recently developed easy and rapid research tool, has been adopted by certain researchers $(14,15)$. In the present study, we used the baculovirus system to produce the biologically active DUSP1 protein and we subsequently examined its effects on cancer cells following incubation with DUSP1 protein.

\section{Materials and methods}

Materials. The template DUSP1 gene was obtained from Open Biosystems/Thermo Fisher Scientific Inc. (Rockford, IL, USA); The BamHI and HindIII restriction enzymes were purchased from New England Biolabs (Ipswich, MA, USA); Taq DNA 
polymerase and T4 ligase were purchased from Takara Bio Inc. (Tokyo, Japan). The plasmid isolation kit, Cellfectin ${ }^{\circledR}$ reagent, the Sf9 cell line, the pFastBacl cloning kit, the ECL kit and growth media (Grace's insect medium, DMEM and fetal bovine serum) were all purchased from Invitrogen (Carlsbad, CA, USA). The anti-His tag antibody was from Tiangen Biotech (Beijing, China; Cat. no. 168-10481); the p-ERK antibody was obtained from Cell Signaling Technology Inc. (Danvers, MA, USA; Cat. no. 2371); the HRP-anti-mouse IgG Fc was purchased from Sigma-Aldrich Inc. (St. Louis, MO, USA; Cat. no. 63134). All other reagents and instruments were provided by ProMab Biotechnologies Inc. (Richmond, VA, USA; Cat. no. 23173).

Cell culture. Sf9 insect cells were cultured in Grace's insect medium containing $10 \%$ fetal bovine serum at $37^{\circ} \mathrm{C}$ without $\mathrm{CO}_{2}$ in Petri dishes. The cells cultures were subculured at a ratio of 1:5 (cells:medium) and the medium was renewed every 2 to 3 days. The cells in suspension were cultured in Grace's insect medium containing 1\% F-68 in a sterile flask and incubated on a shaking table at a speed of $120 \mathrm{rpm}$. Cell density was kept at $1-2 \times 10^{6}$ cells $/ \mathrm{ml}$. Subcultures were prepared twice each week.

Cancer cell lines (A549 human lung cancer cells, HepG2 human hepatoma cells, PC-2 human pancreatic cancer cells, HeLa human cervical carcinoma cells, MCF-7 human breast cancer cells and GC-7901 human gastric cancer cells) were cultured in the Dulbecco's modified Eagle's medium (DMEM) containing $10 \%$ fetal bovine serum (FBS) and $0.05 \mu \mathrm{g} / \mathrm{ml}$ kanamycin at $37^{\circ} \mathrm{C}$ and $5 \%$ atmospheric $\mathrm{CO}_{2}$. Subcultivation was conducted by transferring $20 \%$ of monolayer cells to new growth medium every 2 to 3 days. To examine the effect of DUSP1, the cells were seeded into 6-well plates at a density of $1 \times 10^{6}$ cells/well or on 96 -well plates at a density of $1 \times 10^{5}$ cells/well. Cell monolayers were incubated for $24 \mathrm{~h}$ before the experiment was conducted.

Primer design and PCR amplification. Based on the sequence of the DUSPI template, PCR amplification was performed with Taq DNA polymerase using the following primers: upstream, 5'-CTCGGATCCACCATGGTCATGGAAGTGGG CACCCT-3'; and downstream, 5'-CTCAAGCTTAGTGG TGATGGTGATGATGGCAGCTGGGAGAGGTCGTAAT-3'. Following amplification, a Bam $\mathrm{HI}$ restriction site was added in front of the upstream primer, and a HindIII restriction site and termination codon were added after the downstream primer. PCR was performed under the following conditions: pre-denaturation at $94^{\circ} \mathrm{C}$ for $3 \mathrm{~min} ; 35$ cycles of denaturation at $94^{\circ} \mathrm{C}$ for $45 \mathrm{sec}$, annealing at $55^{\circ} \mathrm{C}$ for $45 \mathrm{sec}$ and extension at $72^{\circ} \mathrm{C}$ for $5 \mathrm{~min}$; final extension at $72^{\circ} \mathrm{C}$ for $7 \mathrm{~min}$. PCR products were separated on a $1 \%$ agarose gel and the target fragment was recovered by gel elution.

Plasmid construction. The PCR fragment of DUSPI was double digested by $\mathrm{BamHI}$ and HindIII at $37^{\circ} \mathrm{C}$ for $2 \mathrm{~h}$ before the target fragment was gel purified on a $1 \%$ agarose gel. The recombinant plasmid, pFastBac1-DUSP1, was constructed by inserting the DUSPI fragment into the pFastBacl vector at the BamHI and HindIII sites, and then transformed into E. coli $\mathrm{DH} 10 \mathrm{Bac}$ cells by incubation at $42^{\circ} \mathrm{C}$ for $90 \mathrm{sec}$ followed by $30 \mathrm{~min}$ on ice incubation. The transformed cells were subsequently recovered in $500 \mathrm{ml} \mathrm{LB}$ medium by shaking and incubation at $37^{\circ} \mathrm{C}$ for $4 \mathrm{~h}$. Positive colonies were screened by plating the recovered cells $(2,20$ and $200 \mu \mathrm{l})$ on LB agar plates containing gentamicin, kanamycin, tetracycline, $\mathrm{X}$-gal and IPTG and incubation at $37^{\circ} \mathrm{C}$ until the appearance of blue or white colonies. A single white clony was picked up and transferred to a new LB agar plate by streaking and incubation for $72 \mathrm{~h}$. Subsequently, a well separated white colony from this plate was used to inoculate $10 \mathrm{ml}$ of liquid LB medium by shaking and incubation for $14 \mathrm{~h}$. Subsequently, the cells were collected for isolation and confirmation of the recombinant bacmid DNA.

Transfection and western blot analysis. When the density of the Sf9 cells reached $70-80 \%$ in the 6 -well culture plate, the recombinant bacmid DNA was transfected into the Sf9 cells using the Cellfectin reagent. If obvious signs of infection were observed under the microscope (Olympus IX70; Olympus, Tokyo, Japan) 5 days after transfection, the supernatant and the cells were collected separately. The supernatant medium contained the P1 generation of the virus. The collected cells were rapidly washed with phosphate-buffer and sonicated on ice in SDS loading buffer $(62.5 \mathrm{mM}$ Tris- $\mathrm{HCl}$ with $\mathrm{pH} 6.8,10 \%$ glycerol, $50 \mathrm{mM}$ DTT, $2 \%$ SDS and $0.025 \%$ bromphenol blue) 3 times (sonication for $3 \mathrm{sec}$ and rest for $3 \mathrm{sec}$ ). The lysates were stored at $-20^{\circ} \mathrm{C}$ until further analysis. Western blot analysis was carried out according to Molecular Cloning: A Laboratory Manual (16).

Expression of DUSP1 protein and sodium dodecyl sulphatepolyacrylamide gel electrophoresis (SDS-PAGE). The amplification of the viruses was carried out according to the Invitrogen protocol. Briefly, the P1 generation of the virus was used to infect the Sf9 cells. Following lysis of the majority of the cells, the supernatant was collected as the P2 generation, which was then used to inoculate $100 \mathrm{ml}$ of the Sf9 cells at a density of $2 \times 10^{6}$ cells $/ \mathrm{ml}$. The mixture was incubated for $72 \mathrm{~h}$ at $27^{\circ} \mathrm{C}$ before the cells were harvested to collect the DUPS1 protein. The harvested cell pellet was resuspended in PBS, sonicated on ice for 99 cycles (sonication for $10 \mathrm{sec}$, rest for $10 \mathrm{sec}$ ) and centrifuged at $12,500 \mathrm{rpm}$ for $25 \mathrm{~min}$. The supernatant was collected and purified by affinity chromatography using a nickel column. The recombinant protein was eluted by gradient imidazole solutions. For SDS-PAGE, $40 \mu \mathrm{l}$ of total protein were added to $10 \mu \mathrm{l} 5 \mathrm{X}$ loading buffer and boiled for $5 \mathrm{~min}$ before the mixture was loaded onto a polyacrylamide gel.

Western blot analysis of p-ERK expression in 6 cancer cell lines. Six cancer cell lines (HepG2, PC-2, HeLa, MCF-7, A549 and GC-7901) were cultured in 6-well plates separately before $1 \mu \mathrm{g} / \mathrm{ml}$ purified DUSP1 protein was added into each well. Each cell line was incubated with DUSP1 for various periods of time $(0,1,2,4,6$ and $8 \mathrm{~h})$. Thereafter, the cells were harvested and lysed in lysis buffer. For western blot analysis of p-ERK, lysate from each cell type at each time point was loaded onto a gel for SDS-PAGE $(20 \mu \mathrm{l} /$ well). Following resolution, the proteins were electrotransferred onto nitrocellulose membranes at a constant current (300 mA/70 min) and on ice. The membranes were then blocked in TBST containing 5\% dried fat-free milk for $2 \mathrm{~h}$ at room temperature, followed by $2 \mathrm{~h}$ of incuba- 


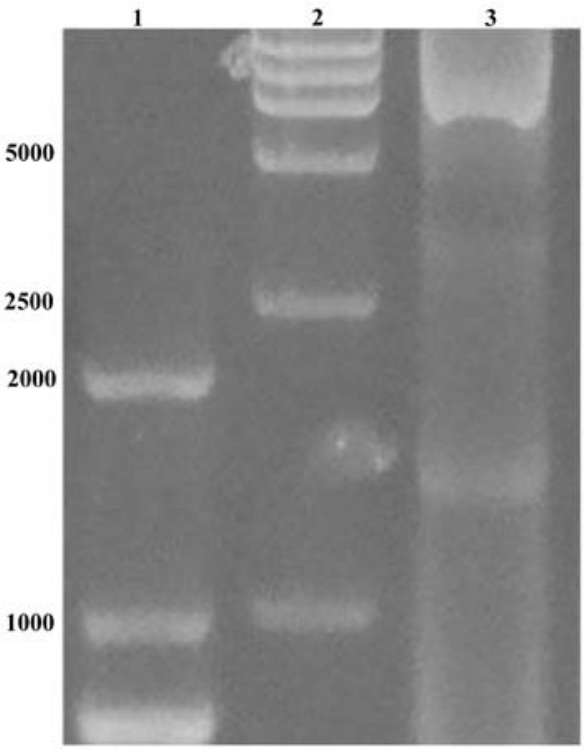

Figure 1. Agarose gel analysis of the DUSP1 PCR product. Lane 1, $2 \mathrm{~kb}$ marker; lane 2, $15 \mathrm{~kb}$ marker; lane 3, amplified PCR product of the DUSP1 gene $(\sim 1.4 \mathrm{~kb})$. DUSP1, dual specificity phosphatase 1 .

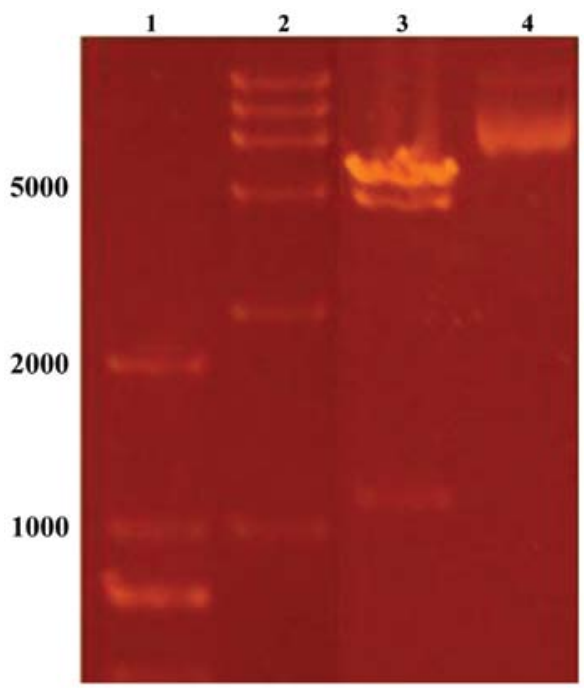

Figure 2. Restriction analysis of the DUSP1 plasmid construct. Lane 1, $2 \mathrm{~kb}$ marker; lane 2, $15 \mathrm{~kb}$ marker; lane 3, digestd plasmid construct containing viral DNA DUSP1; lane 4, undigested recombinant virus DNA.

tion at room temperature with the primary antibody (p-ERK antibody, 1:500; or $\beta$-actin antibody, 1:4x104). The membranes were subsequently washed 3 times in TBST and incubated for $1 \mathrm{~h}$ at room temperature with HRP-labeled secondary antibody $\left(1: 2 \times 10^{4}\right)$. Following extensive washes, the signals were visualized using the ECL kit according to the manufacturer's instructions.

Analysis of the growth of HeLa cells. When the cells reached $40-50 \%$ confluency in monolayer in the 96-well plate, DUSP1 protein was added to each well (apart from the blank control) at a final concentration of $1 \mu \mathrm{g} / \mathrm{ml}$. The cells were then incubated for various periods of time $(0,12,24,36,48,60,72,84$ and $96 \mathrm{~h}$ ) before they were collected to count the cell number.

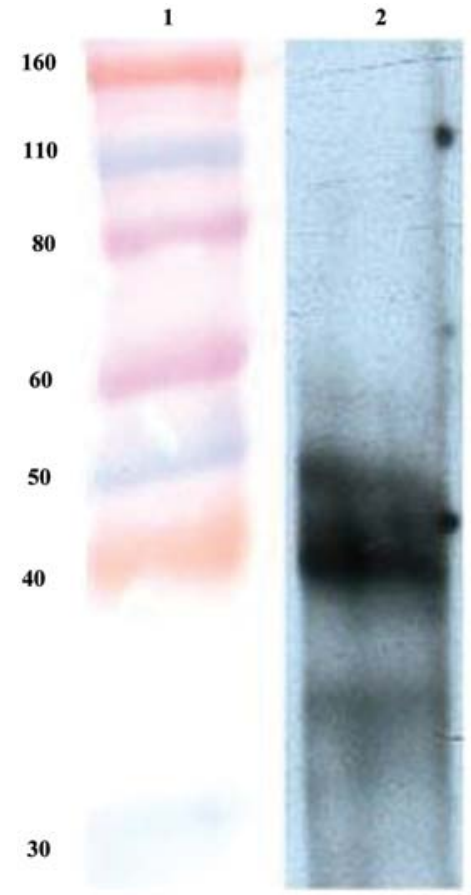

Figure 3. Western blot analysis of DUSP1. Lane 1, protein marker; lane 2, DUSP1 ( 40 kDa). DUSP1, dual specificity phosphatase 1.

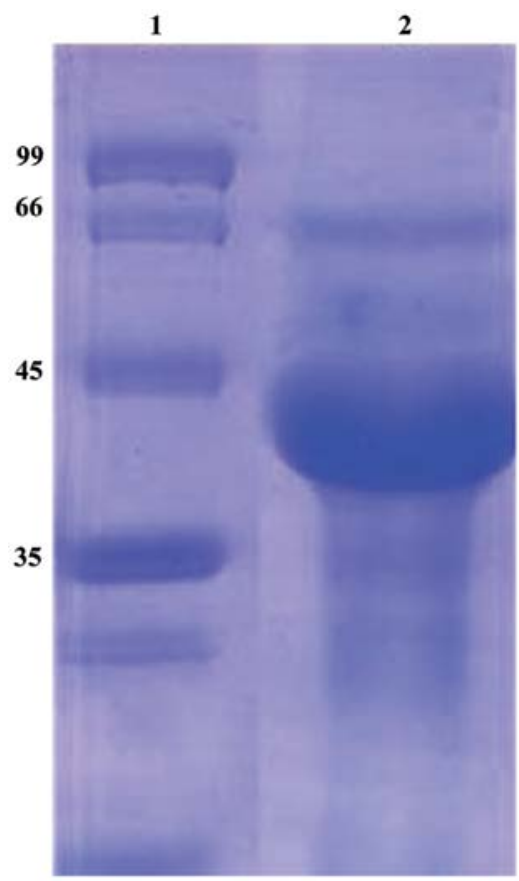

Figure 4. SDS-PAGE of the purified DUSP1 protein. Lane 1, protein marker; lane 2, Ni-NTA agarose-purified DUSP1 protein. DUSP1, dual specificity phosphatase 1.

Quadruplets were performed for each data point and the average value was used to plot the growth curve.

\section{Results}

Amplification of the target fragment. The amplified PCR product (Fig. 1) was verified by agarose gel electrophoresis. The segment size was in line with expectations. 
Table I. Effect of DUSP1 on the growth rate of HeLa cells.

\begin{tabular}{lcccccccc}
\hline Time (h) & 0 & 12 & 24 & 36 & 48 & 60 & 72 & 84 \\
\hline HeLa $\left(10^{4}\right)$ & 16.5 & 26.5 & 33 & 45.5 & 50.75 & 67.5 & 106.5 & 107.5 \\
HeLa + DUSP1 $\left(10^{4}\right)$ & 16.5 & 21 & 31 & 40.75 & 49.5 & 64.7 & $\begin{array}{c}108 \\
77.5\end{array}$ & 88 \\
\hline
\end{tabular}

The cell number in each group was tracked at various time points following exposure to DUSP1. DUSP1, dual specificity phosphatase 1 .
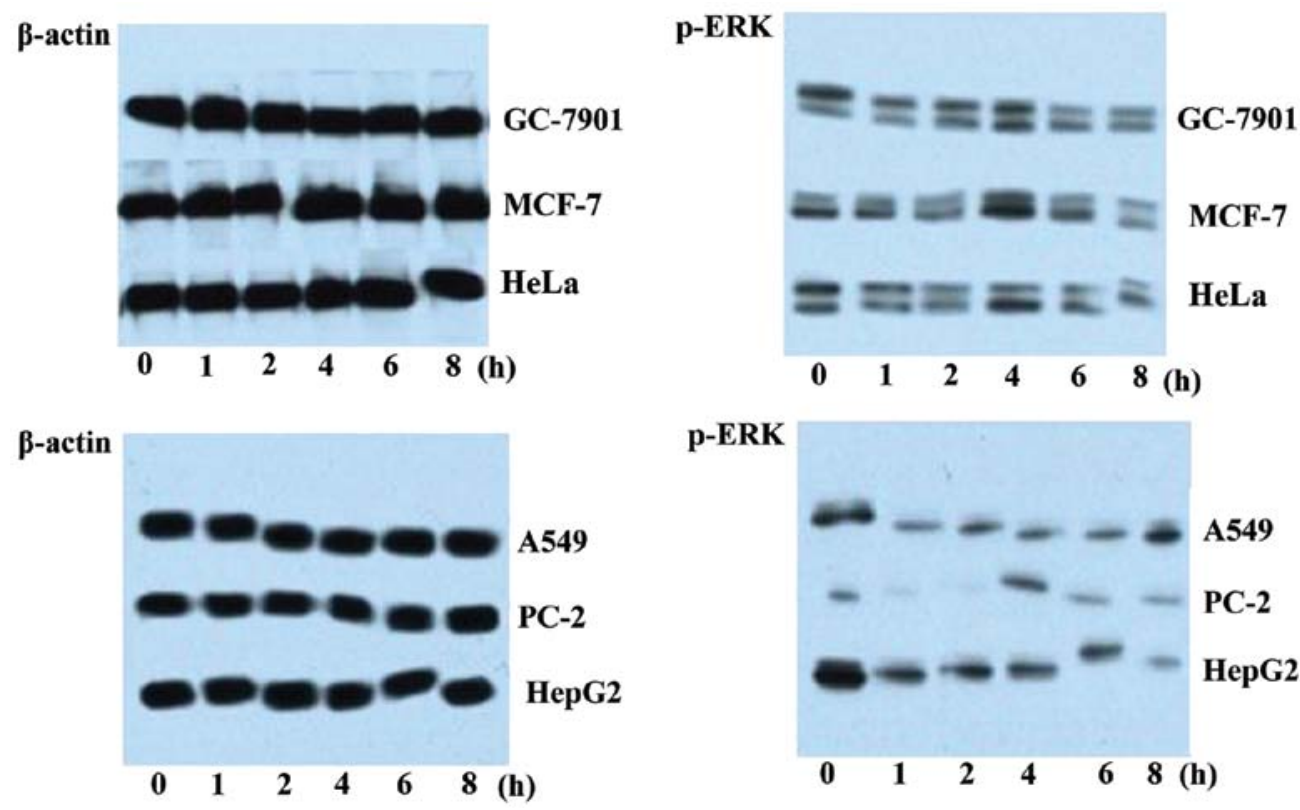

Figure 5. Effect of exposure to DUSP1 on the expression of phosphorylated extracellular signal-regulated kinase (p-ERK) in GC-7901, MCF-7, HeLa, A549, PC-2 and HepG2 cancer cell lines. Cancer cells were exposed to purified DUSP1 for 0,1,2,4,6 and $8 \mathrm{~h}$ before they were harvested and analyzed by western blot analysis. $\beta$-actin was used as the internal control. DUSP1, dual specificity phosphatase 1 .

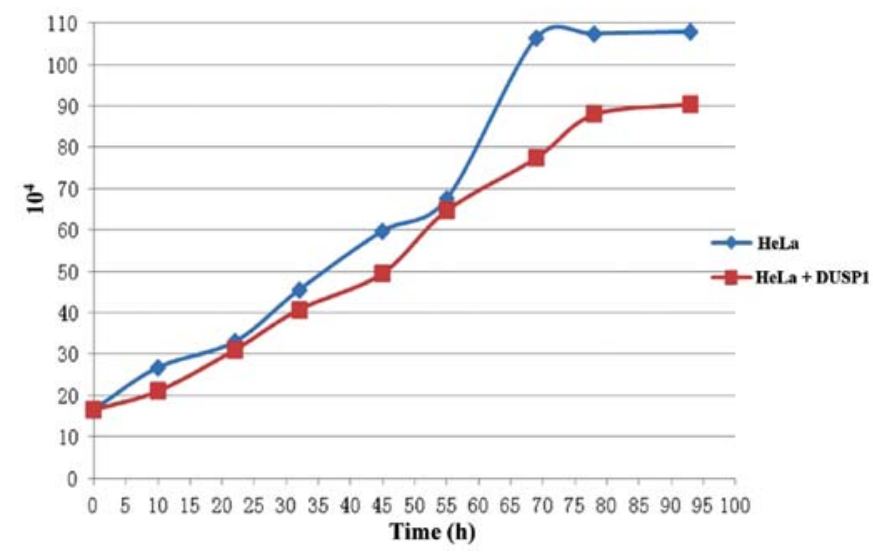

Figure 6. Effect of DUSP1 on the growth rate of human cervical cancer cells (HeLa). DUSP1, dual specificity phosphatase 1.

Plasmid constructs. The DUSPI gene was inserted at the Bacmid locus of the pFastBac1 vector. Since the $\alpha$-complementarity was broken, the bacterium bearing the resulting plasmid construct produced white colonies on solid agar plates containing gentamicin (Gm), kanamycin (Kan), tetracycline (Tet) and $\mathrm{X}$-gal. Plasmids isolated from these white colonies were double digested with BamHI and HindIII, and the resultant fragment produced the expected size of the DUSPI gene (Fig. 2) when analyzed on an agarose gel. The gene sequence was further confirmed through sequencing of the recombinant plasmid.

Western blot analysis. Five days after transfection of the recombinant plasmid, the Sf9 cells in the middle of 6-well culture plate appeared darker than the ones on the edge when observed under an inverted microscope. These darker cells showing apparent co-transfection were harvested and lysed for western blot analysis. The result revealed that DUSP1 was successfully expressed in the Sf9 cells (Fig. 3).

SDS-PAGE. The lysate of Sf9 cells containing DUSP1 protein was purified by Ni-affinity chromatography. The purified products were examined by SDS-PAGE. A prominent band with the identical size of DUSP1 was found in the lysate (Fig. 4).

Effect of DUSP1 on the level of p-ERK in cancer cells. The expression of p-ERK in the 6 different cancer cell lines as analyzed by western blot analysis following exposure to DUSP1 for various periods of time (Fig. 5). Compared to the $\beta$-actin level which remained constant following exposure to DUSP1, the amount of p-ERK markedly decreased after $1 \mathrm{~h}$, indicating that DUSP1 suppressed the expression of p-ERK in all 6 cancer cell lines. 
Effect of DUSP1 on the proliferation rate of cancer cells. As shown in Table I and Fig. 6, DUSP1 affected the proliferation rate of the cancer cells. For example, the HeLa cells showed a much smoother growth curve when exposed to DUSP1, lacking the apparent pattern of the exponential phase. The capability of binary fission was also reduced when the cells were examined under a microscope, suggesting that DUSP1 suppressed the proliferation of the cancer cells.

\section{Discussion}

MAPKs, including ERK, JNK and p38, play critical roles in the regulation of cell growth, differentiation and the control of cellular responses to cytokines and stress (17). The MAPK-ERK signaling pathway plays an important role in cell metabolism and has attracted widespread attention in recent years (18). The prototype of this family, DUSP1 (encoding the protein MKP-1), regulates the activation of ERK, JNK and p38, and the alteration of DUSP1 expression has been associated with malignant progression in a various types of cancer (19). DUSP1 protein suppresses the activity of MAPK-ERK through dephosphorylation. Since DUSP1 also dephosphorylates p-ERK, the mechanisms through which DUSP1 modulates the MAPK-ERK pathway are not yet clear. Therefore, a better understanding of the specific mechanisms responsible for the regulation of DUSP1 by p-ERK and the functional consequences of this regulation is required (20).

The baculovirus protein expression system is a newly discovered superior post-translational modification system compared to the traditional E. coli expression system, and it produces large quantities of protein with near-natural protein functions. In this study, we expressed DUSP1 using this improved expression tool and demonstrated that DUSP1 suppressed the proliferation of HeLa cells, possibly through the regulation of the MAPK-ERK signaling pathway. In addition to the possibility of the regulation of DUSP1 by replicationindependent mechanisms in HeLa cells, it is plausible that DUSP1 expression is regulated by other replication-dependent mechanisms (21). Overall, our data demonstrate that DUSP1 inhibits the proliferation of human cervical cancer cells and provide valuable resources which will consitute the basis of further investigations regarding the factors responsible for the proliferation of cancer cells.

\section{Acknowledgements}

The present study was supported by the Ministry of Agriculture '948' project (2013-Z58).

\section{References}

1. Hammer M, Mages J, Dietrich H, et al: Dual specific city hosphatase 1 (DUSP1) regulates a subset of LPS-induced genes and protects mice from lethal endotoxin shock. J Exp Med 1: 15-20, 2006.
2. Moncho-Amor V, Ibañez de Cáceres I, Bandres E, et al: DUSP1/MKP1 promotes angiogenesis, invasion and metastasis in non-small-cell lung cancer. Oncogene 30: 668-678, 2011.

3. Chen CC, Hardy DB and Mendelson CR: Progesterone receptor inhibits proliferation of human breast cancer cells via induction of MAPK phosphatase 1 (MKP-1/ DUSP1). J Biol Chem 286: 43091-43102, 2011.

4. Liu C, Shi Y, Du Y, et al: Dual-specificity phosphatase1 DUSP1 protects over activation of hypoxia-inducible factor 1 through inactivating ERK MAPK. Exp Cell Res 309: 410-418, 2005.

5. Wu W, Pew T, Zou M, Pang D and Conzen SD: Glucocorticoid receptor-induced mapk phosphatase-1(MPK-1) expression inhibits paclitaxel-associated MAPK activation and contributes to breast cancer cell survival. J Biol Chem 280: 4117-4124, 2005.

6. Purwana IN, Kanasaki H, Mijiddorj T, Oride A and Miyazaki K: Induction of dual-specificity phosphatase 1 (DUSP1) by pulsatile gonadotropin-releasing hormone stimulation: role for gonadotropin subunit expression in mouse pituitary LbetaT2 cells. Biol Reprod 84: 996-1004, 2011.

7. Nyati MK, Feng FY, Maheshwari D, et al: Ataxia telangiectasia mutated down-regulates phosphor-extracellular signal-regulated kinase $1 / 2$ via activation of MKP-1 in response to radiation. Cancer Res 66: 11554-11559, 2006.

8. Wang J, Yin DP, Liu YX, Baer R and Yin Y: Dual specificity phosphatase 1/CL100 is a direct transcriptional target of E2F-1 in the apoptotic response to oxidative stress. Cancer Res 67: 6737-6744, 2007.

9. Jacob A, Rajan D, Pathickal B, et al: The inhibitory effect of ghrelin on sepsis-induced inflammation is mediated by the MAPK phosphatase-1. Int J Mol Med 25: 159-164, 2010.

10. Calvisi DF, Pinna F, Meloni F, et al: Dual-specificity phosphatase 1 ubiquitination in extracellular signal-regulated kinase-mediated control of growth in human hepatocellular carcinoma. Cancer Res 68: 4191-4200, 2008.

11. Liu YX, Wang J, Guo J, et al: DUSP1 is controlled by p53 during the cellular response to oxidative stress. Mol Cancer Res 6: 624-633, 2008.

12. Hägg S, Alserius T, Noori P, et al: Blood levels of dual-specificity phosphatase-1 independently predict risk for post-operative morbidities causing prolonged hospitalization after coronary artery bypass grafting. Int J Mol Med 27: 851-857, 2011.

13. Gómez-Sebastián S, Nuñez MC, Garaicoechea L, Alvarado C, Mozgovoj M, Lasa R, Kahl A, Wigdorovitz A, Parreño V and Escribano JM: Rotavirus A-specific single-domain antibodies produced in baculovirus-infected insect larvae are protective in vivo. BMC Biotechnol 12: 59, 2012.

14. Farinha-Arcieri LE, Porchia BM, Carromeu C, Simabuco FM, et al: Expression and purification of a recombinant adenovirus fiber knob in a baculovirus system. Intervirology 51: 189-195, 2008.

15. Cha HJ, Dalal NG, Vakharia VN and Bentley WE: Expression and purification of human interleukin-2 simplified as a fusion with green fluorescent protein in suspended Sf-9 insect cells. J Biotechnol 69: 9-17, 1999

16. Maniatis T: Molecular cloning: a laboratory manual. Cold Spring Harbor Laboratory Press, New York, pp403-410, 1992.

17. Chang L and Karin M: Mammalian MAP kinase signalling cascades. Nature 410: 37-40, 2001.

18. Fey D, Croucher DR, Kolch W and Kholodenko BN: Crosstalk and signaling switches in mitogen-activated protein kinase cascades. Front Physiol 3: 355,2012 .

19. Wu GS: Role of mitogen-activated protein kinase phosphatases (MKPs) in cancer. Cancer Metastasis Rev 26: 579-585, 2007.

20. Keyse SM: Dual-specificity MAP kinase phosphatases (MKPs) and cancer. Cancer Metastasis Rev 27: 253-261, 2008.

21. Qin Z, Dai L, Defee M, Findlay VJ, Watson DK, Toole BP, Cameron J, Peruzzi F, Kirkwood K and Parsons C: Kaposi's sarcoma-associated herpesvirus suppression of DUSP1 facilitates cellular pathogenesis following de novo infection. J Virol 87: 621-635, 2013. 